\title{
In Situ Scanning Probe Microscopy Nanomechanical Testing
}

AUTHOR(S):

Li, Xiaodong; Chasiotis, Ioannis; Kitamura, Takayuki

\section{CITATION:}

Li, Xiaodong ...[et al]. In Situ Scanning Probe Microscopy

Nanomechanical Testing. MRS BULLETIN 2010, 35(5): 361-367

ISSUE DATE:

2010

URL:

http://hdl.handle.net/2433/148415

RIGHT:

(C) 2009, Materials Research Societyiety 
In Situ Scanning Probe Microscopy Nesting

\title{
Xiaodong Li, loannis Chasiotis, and Takayuki Kitamura
}

\begin{abstract}
Scanning probe microscopy (SPM) has undergone rapid advancements since its invention almost three decades ago. Applications have been extended from topographical imaging to the measurement of magnetic fields, frictional forces, electric potentials, capacitance, current flow, piezoelectric response and temperature (to name a few) of inorganic and organic materials, as well as biological entities. Here, we limit our focus to mechanical characterization by taking advantage of the unique imaging and force/displacement sensing capabilities of SPM. This article presents state-of-theart in situ SPM nanomechanical testing methods spanning (1) probing the mechanical properties of individual one-dimensional nanostructures; (2) mapping local, nanoscale strain fields, fracture, and wear damage of nanostructured heterogeneous materials; and (3) measuring the interfacial strength of nanostructures. The article highlights several novel SPM nanomechanical testing methods, which are expected to lead to further advancements in nanoscale mechanical testing and instrumentation toward the exploration and fundamental understanding of mechanical property size effects in nanomaterials.
\end{abstract}

\section{Introduction}

Recent developments in science and engineering have provided the capability to fabricate and control structures and materials at the nanoscale. The functionality and reliability of micro/nanodevices are often determined by the mechanical properties of the individual nanostructures and the different materials comprising these devices. In this regard, it has been shown that the mechanical properties of structures and materials are size-dependent. Since nanostructures and nanostructured materials often exhibit unique properties at ultrasmall scales, unlike their counterparts at the macroscale, the successful transition of nanostructures and nanostructured materials to practical applications requires their mechanical characterization at the nanoscale. ${ }^{1-7}$ To perform such nanoscale mechanical experiments, an instrument needs to have two basic functions: to "see" a nanostructure and deform/fracture the nanostructure in situ. Scanning probe microscopy (SPM) detects interactions between a nanoscale probe and a nearby surface in order to map the surface morphology and its properties.8,9 By taking advantage of SPM's high spatial resolution, a micro/nanomechanical tester can be integrated with an SPM to perform mechanical experiments on nanostructured bulk materials where the sample surface is imaged in situ by the SPM. Possessing both nanoscale force and displacement sensing capabilities, atomic force microscopy (AFM) and its variants are also able to directly probe the mechanical properties of nanostructures and nanostructured bulk materials. As will be demonstrated in the following selected examples, SPM provides insights into the mechanical behavior of nanostructures and nanostructured bulk materials, which helps us to not only measure their mechanical properties but also to understand the fundamental mechanisms that govern the mechanical property size effects in nanomaterials.

\section{Probing the Mechanical Properties of One-Dimensional Nanostructures}

The ultrasmall dimensions of onedimensional (1D) nanostructures (e.g., nanotubes, nanowires, and nanofibers) have posed great challenges to existing instruments, methodologies, and well-established theories. AFM has been proven to be a powerful tool for measuring the mechanical properties of isolated individual 1D nanostructures. Both AFM contact and lateralforce modes have been used to bend 1D nanostructures, such as carbon nanotubes and nanowires of various materials in either three-point bending or cantilever configurations. ${ }^{10-19}$ The corresponding AFM tip force-deflection curves contain information about the deformation behavior of nanostructures from which the mechanical properties, such as the elastic modulus and the yield strength, can be derived. Postexperimental in situ imaging provides the ability to verify that the test has been performed in the anticipated location, which maximizes the reliability of data and aids in the explanation of unexpected results.

Here, we use an AFM three-point bending test to show the importance of calibration procedures for nanoscale measurements. In an AFM three-point bending test, the measured total displacement consists of two components: the deflection of the cantilever and the deflection of the nanostructure. To obtain only the latter, the cantilever deflection must be determined. On the other hand, to avoid penetration of the AFM tip into the nanostructure during a bending test, the maximum applied load, below which negligible penetration occurs, should be identified. Therefore, a careful calibration procedure is suggested before each test. As an example, Figure 1 shows the calibration procedure for an AFM three-point bending test on a $\mathrm{SiO}_{2}$ nanowire. ${ }^{14}$ An AFM indentation experiment was first performed on a $\mathrm{SiO}_{2}$ nanowire on silicon substrate (Figure $1 \mathrm{a}-1 \mathrm{~b})$. At $4 \mu \mathrm{N}$ indentation force, the penetration of the AFM tip into the nanowire (i.e., the nanowire deflection shown in Figure $1 b$ ) was within the range of $\pm 1 \mathrm{~nm}$ after subtracting the cantilever deflection. This force was the maximum applied load used in three-point bending tests. The AFM cantilever versus the vertical piezo position curve for a suspended $\mathrm{SiO}_{2}$ nanowire under an applied load of $3.3 \mu \mathrm{N}$ and until fracture-failure and the corresponding 


\section{In Situ Scanning Probe Microscopy Nanomechanical Testing}
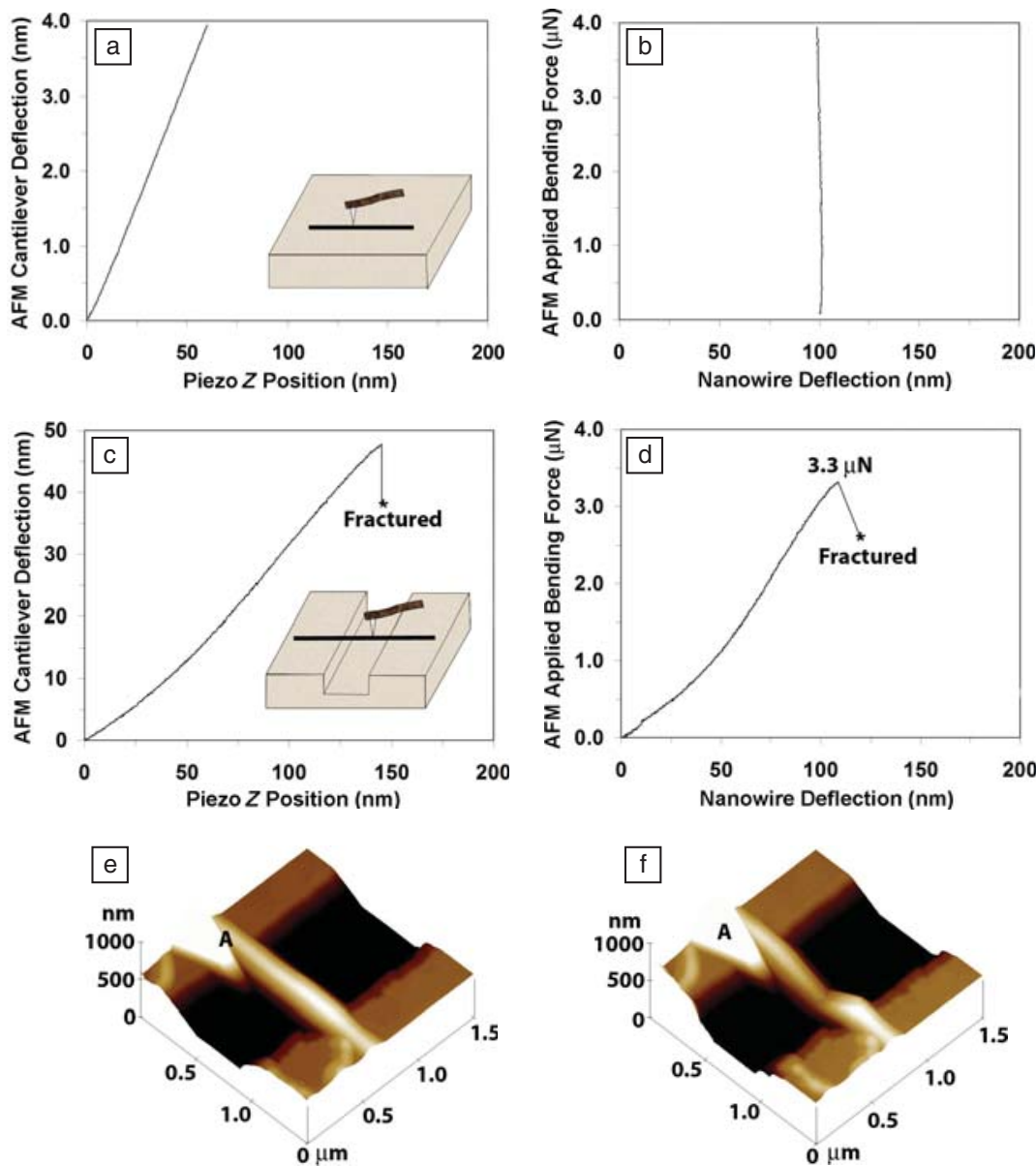

Figure 1. (a) Cantilever and (b) nanowire deflections obtained by three-point bending using an atomic force microscopy (AFM) tip to directly indent a $\mathrm{SiO}_{2}$ nanowire on a solid $\mathrm{Si}$ substrate. (c) Cantilever and (d) nanowire deflections obtained from a suspended $\mathrm{SiO}_{2}$ nanowire. (e) and (f) 3D AFM images showing the suspended $\mathrm{SiO}_{2}$ nanowire morphology before and after three-point bending corresponding to (c) and (d), respectively. The nanowire fractured in a brittle manner at a bending force of $3.3 \mu \mathrm{N}$. Note: the triangular notch-like area labeled "A" was a piece dirt. ${ }^{14}$

applied load versus nanowire deflection relationship are shown in Figure 1c and $1 \mathrm{~d}$, respectively. The slope of the initial linear portion of the curve in Figure $1 \mathrm{~d}$ was calculated and treated as the spring constant of the nanowire. The plastic deformation and nanowire fracture mechanics at the center of the wire can be further studied by analyzing the nonlinear portion of the load-deflection curve (Figure 1d) and the AFM image of the fractured wire (Figure 1f).

AFM also was used to directly bend individual, well-aligned, 1D nanostructures grown vertically on a solid substrate without destruction or manipulation of the nanostructures. By scanning the vertically aligned $\mathrm{ZnO}$ nanowires, the lateral force-deflection data were acquired to derive the elastic modulus of the individual $\mathrm{ZnO}$ nanowires. ${ }^{20}$ This method offers fast measurement of the elastic modulus of nanostructures of different sizes..$^{20,21}$ Another approach is to cleave the substrate with well-aligned nanowires along the wire growth axis to realize an array of cantilever nanowires at the cleaved edge for AFM bending tests. ${ }^{22}$ High bending load on such cantilever nanowires resulted in fracture of the wires from their fixed ends, allowing their fracture strength to be measured.

The elastic properties of $\mathrm{ZnO}$ nanobelts were recently investigated using the AFM nanoindentation technique. ${ }^{23,24}$ The AFM probe was used to image/locate individual $\mathrm{ZnO}$ nanobelts sitting on a rigid substrate and then to indent them in situ. The elastic moduli of nanobelts with different widthto-thickness ratios were derived from the indentation load-displacement curves based on the Hertzian contact theory. The elastic modulus was found to decrease from about $100 \mathrm{GPa}$ to about $10 \mathrm{GPa}$, with increasing width-to-thickness ratio.

AFM is unique in terms of studying the mechanical behavior of biomaterials. ${ }^{25-28}$ The electron beam radiation and the vacuum in an electron microscope may alter the surface structure of biomaterials, thus affecting their mechanical properties. It has been shown that the surface structure and the surface stress may influence the mechanical properties of nanostructures. ${ }^{29,30}$ The AFM can be operated in a temperature- and humidity-controlled chamber, avoiding the absorption of surface molecules (for instance $\mathrm{H}_{2} \mathrm{O}$ ) and the electron beam radiation on the sample surface that would occur in an electron microscope vacuum chamber. The following example (Figure 2) illustrates how an AFM provides unique insights into the deformation behavior of polyaniline(PANI-) coated tobacco mosaic virus nanotubes that could not be studied otherwise. $^{27}$ A typical indentation curve on a PANI-coated tobacco mosaic virus nanotube can be divided into four stages (I, II, III, and IV). In Stage I, as the AFM tip approached the sample surface from $100 \mathrm{~nm}$ above (not fully shown), a sudden snap-in occurred due to the adhesive force between the tip and sample surface, and then the AFM was brought into contact with the sample. As the tip continued to press against the sample surface within a $5 \mathrm{~nm}$ range (Stage II), the total force exerted on the sample increased linearly, followed by a nonlinear response shown in Stage III. The inner cavity of the nanotube was further squeezed against the $\mathrm{Si}$ wafer shown in Stage IV, where the substrate effect became appreciable.

Finally, contact-resonance AFM provides new paths to measure the elastic modulus of nanowires in both radial and lateral orientations. The radial wire modulus can be determined from the change in resonance frequency that occurs when the probe tip is brought into contact with the wire, whereas the modulus along the wire length is obtained through the frictional force on the AFM cantilever. ${ }^{31}$

\section{Mapping Local Nanoscale Strain Fields, Fracture, and Wear Damage}

Quantitative data of deformation and strain are difficult to obtain at the nanoscale, since, for the imaging domains commonly used with an AFM, such deformations in brittle and quasi-brittle materials are only of the order of 10-20 nm. ${ }^{8}$ Imperative prerequisites to obtain repeatable and accurate surface profiles by AFM are the linearization of the piezoelectric scanner and the 


\section{In Situ Scanning Probe Microscopy Nanomechanical Testing}
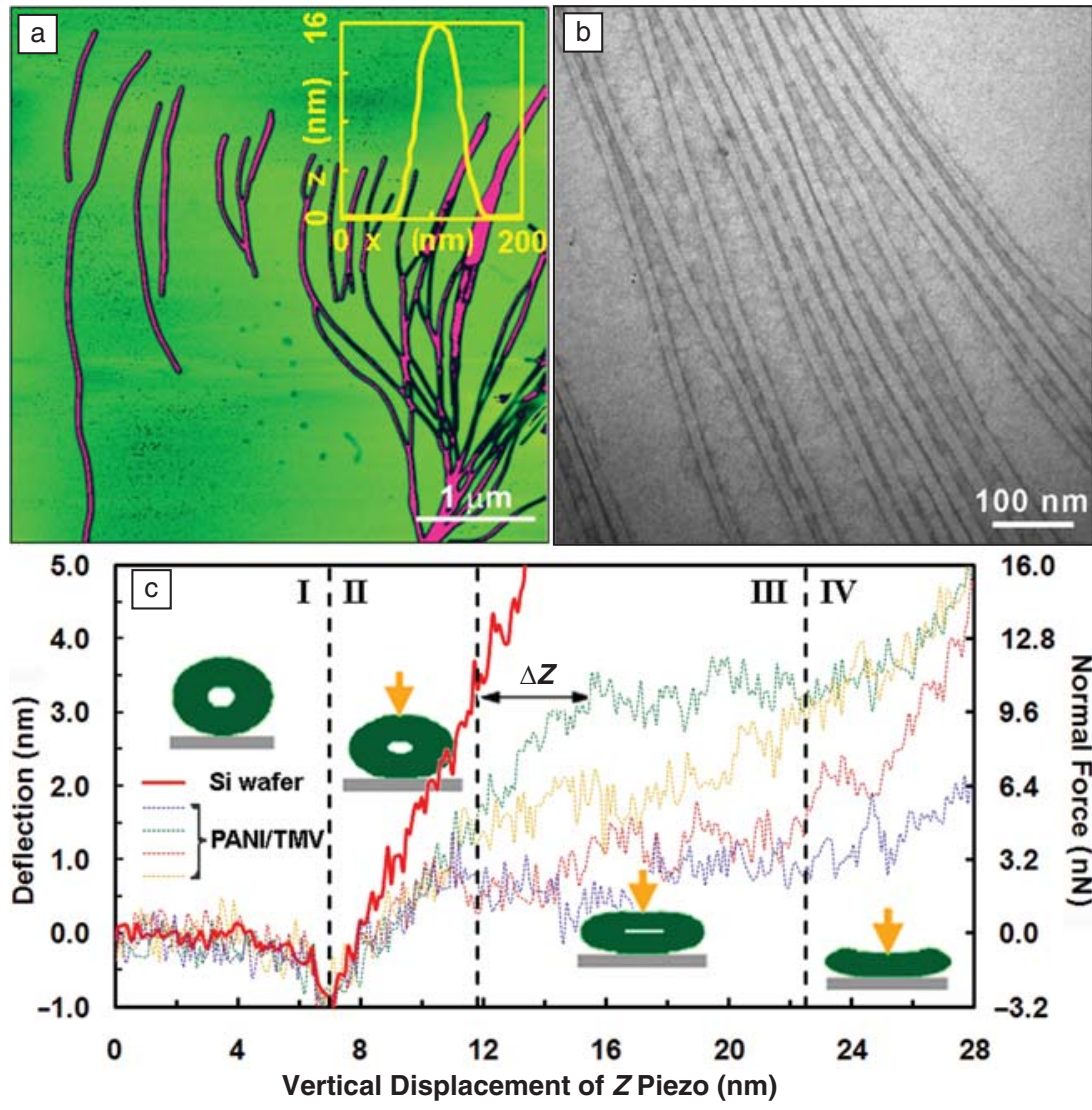

Figure 2. (a) Atomic force microscopy height image of polyaniline (PANI)/ tobacco mosaic virus (TMV) nanotubes on a Si wafer. The inset profile shows the average height of $\sim 15.3 \mathrm{~nm}$. (b) Transmission electron microscopy image of well-dispersed PANI/TMV nanotubes. (c) Plot of cantilever deflection versus vertical displacement of the piezo. The symbol $\Delta Z$ represents the sensitivity. The indentation depth is the difference between the Si wafer and the loading curve of a PANI/TMV tube. The solid red curve was obtained from the indentation on a Si wafer, while the other dashed curves were from the indentations on a PANI/TMV tube at different locations. The cartoons show the cross-sectional view of the indentation process. ${ }^{27}$

construction of stable apparatuses for sample loading. ${ }^{32}$ With these in place, the ability to resolve local deformations has been harnessed to determine displacement fields in amorphous diamond ${ }^{33}$ and polycrystalline silicon 8,34 thin films in order to calculate their mechanical properties.

Topographic AFM measurements compared at different loads by the use of digital image correlation (DIC) have been shown to be quantitatively reliable in solving inverse problems in mechanics of materials, such as the inverse hole problem, where only one displacement field near a circular perforation in a thin film suffices to determine simultaneously the elastic modulus and the Poisson's ratio of a material. ${ }^{35}$ "Inverse problems" start with the solution of the boundary value problem in terms of displacements to back-calculate the elastic material properties. In such experiments, the displacement and its spatial resolution are of the order of 1-2 $\mathrm{nm}$. In polycrys- talline materials, the solution to the spatial resolution problem is meaningful only if the grain size is much smaller than the circular perforation (i.e., for representative volume elements [the smallest material element for which a specific mechanical property is the same as the bulk material] significantly smaller than the geometric features of a specimen). The AFM/DIC method was applied to determine the minimum representative volume element of polycrystalline silicon thin films with columnar grain structure, and the measurements were very close to the theoretical calculations. ${ }^{36}$ The spatial resolution in mechanical strain measurements is well suited to study heterogeneous materials, such as nanocomposites, where strain localization effects in brittle matrices have been resolved, as shown in Figure 3.37

The local nature of AFM measurements is very appropriate to interrogate the problem of fracture of brittle and quasi-brittle

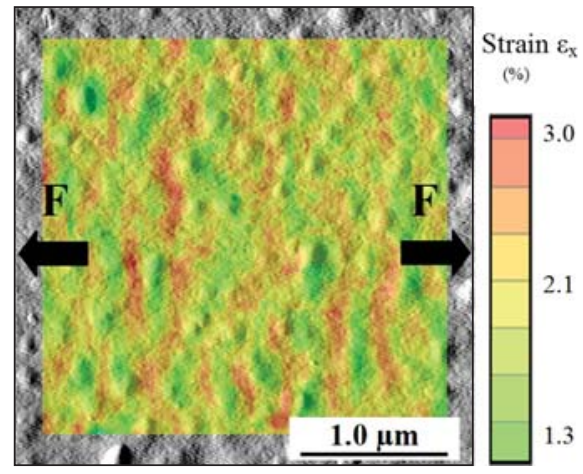

Figure 3. Local strain distribution in a nanocomposite with $100 \mathrm{~nm}$ silica subjected to $1 \%$ macroscale strain. ${ }^{37} \mathrm{~F}$, force.

materials by investigating the vicinity of cracks, propagating in subcritical conditions, in a qualitative and quantitative manner. Qualitatively, nanoscale observations of crack growth in glasses have pointed to a significant region of $\sim 50 \mathrm{~nm}$ near a crack tip, ${ }^{38}$ where the linearly elastic fracture mechanics asymptotic solution did not hold, contrary to the general notion for brittle materials. More recently, AFM observations of subcritical crack growth in glasses and aluminosilicate glasses ${ }^{39,40}$ pointed to cavity formation and coalescence in front of a crack tip with variable velocities $\left(\sim 10^{-10}-10^{-12} \mathrm{~m} / \mathrm{s}\right)$ in a manner that is reminiscent of macroscale ductile fracture. The imaging frequency of an AFM in its most common implementation does not exceed 5-10 Hz, but the acquisition of data at extremely slow crack velocities and at low stress intensity factors is possible. ${ }^{39,40}$ Such studies have been corroborated with molecular dynamics predictions, but further work is needed to link the surface records from AFM with bulk fracture mechanisms. In ductile materials, such studies are more difficult, as time-dependent processes at the crack process zone change the surface landscape, thus, limiting the use of an AFM. Instead, a different adaptation of the AFM has been used to perform nanoindentation experiments near the crack tip to quantify local yielding and hardening. ${ }^{41}$

Measurements of the displacement field in the vicinity of sharp cracks in thin films also have been made possible by the AFM/DIC method. ${ }^{42,43}$ Two micron-thick polycrystalline silicon freestanding thin films with embedded mathematically sharp cracks were subjected to in-plane mode I loading, and the strain fields near the crack tip were obtained by AFM/DIC measurements that compared very well with 2D finite element calculations, as illustrated in Figure $4 .^{42}$ These experiments also revealed a process of subcritical crack 


\section{In Situ Scanning Probe Microscopy Nanomechanical Testing}

growth, different from that discussed before in connection with amorphous glasses, where a crack propagating in a regime of tougher grain boundaries and randomly oriented submicron-scale grains can be arrested and restarted under monotonically increasing far field loading. In the AFM experiments shown in Figure 4, no void nucleation and coalescence was observed, as in the case of glasses, which implies that this mode of fracture is exclusive to amorphous ceramics.

The AFM/DIC method also has been used to measure the local, nanoscale deformation in polymeric thin films and advanced metal evaporated magnetic tapes. 44,45 The deformation in polymeric thin films was nonuniform, and high strain gradients were present at the nanoscale. For metal-evaporated magnetic tapes, cracking started at the strain transition sites, where a large strain gradient existed. The strain was redistributed after crack formation. ${ }^{45}$

A recent study showed that the initiation and development of nanowear, which is usually hard to detect directly from AFM topographical images, can be efficiently detected by monitoring the change in the DIC correlation coefficient from AFM images in the wear-scar zone. ${ }^{46}$ The DIC correlation coefficient describes the convergence of the calculated displacements to the measured ones. The linear relationship between the correlation coefficient and the wear depth can be used to quantify the extent of nanowear damage. The DIC correlation coefficient is more sensitive in visualizing local wear at the nanoscale than the commonly used surface roughness. For example, DIC showed that nanowear of gold coatings is dominated by material removal without any plastic deformation.

\section{Interfacial Strength of Nanostructures}

Since micro/nanodevices often are composed of different materials at the nanoscale, they intrinsically include interfaces, which are the most favorable sites for mechanical failure due to the low adhesion and high stress concentrations caused by mechanical property and microstructure mismatches. For example, the theory of elasticity predicts infinite stress at edges where the interface meets a free surface. Because an AFM can precisely apply ultralight forces at any location and simultaneously sense small displacements, it can be a key instrument in mechanically testing nanocomponents.

Recently, AFM was used to study the interfacial strength between a submicron chromium dot and its silicon substrate. ${ }^{47}$ The dot was removed by an AFM diamond tetrahedral-shaped tip, which was first engaged near the dot and then was dragged over the dot, as shown in Figure 5. The delamination area was imaged in situ after the test. The critical load at which delamination occurred together with the delamination area were used to measure the interfacial strength. It has been shown to be difficult to measure the interface strength of a dot made of a relatively soft material (e.g., $\mathrm{Cu}$ ), because the AFM tip may penetrate the dot, resulting in plastic deformation. In such a case, an overlayer of a

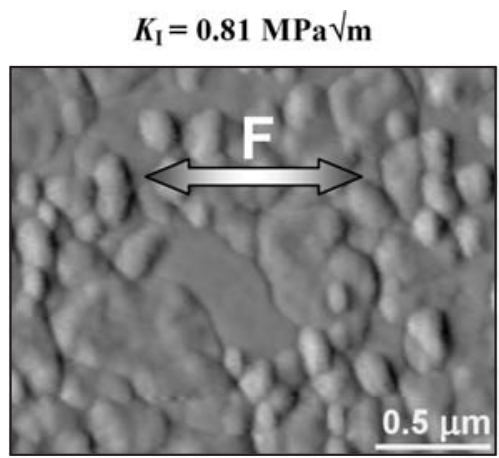

b

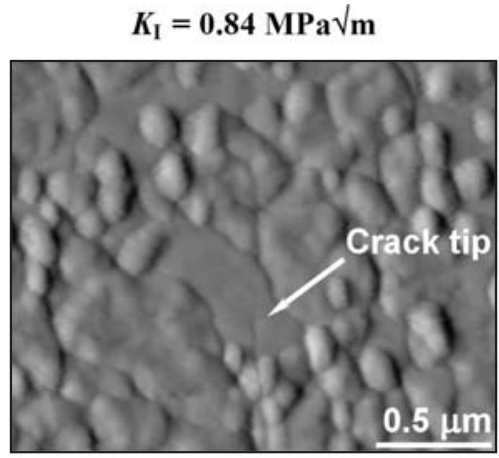

c

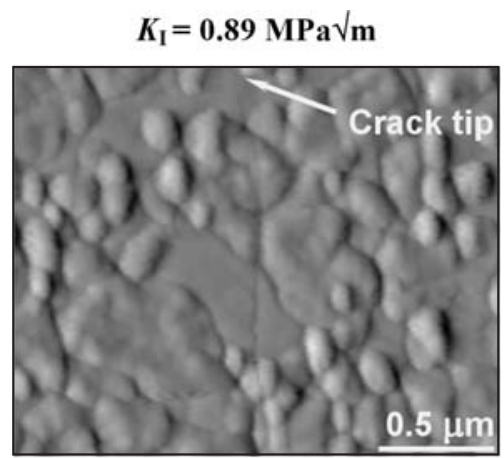

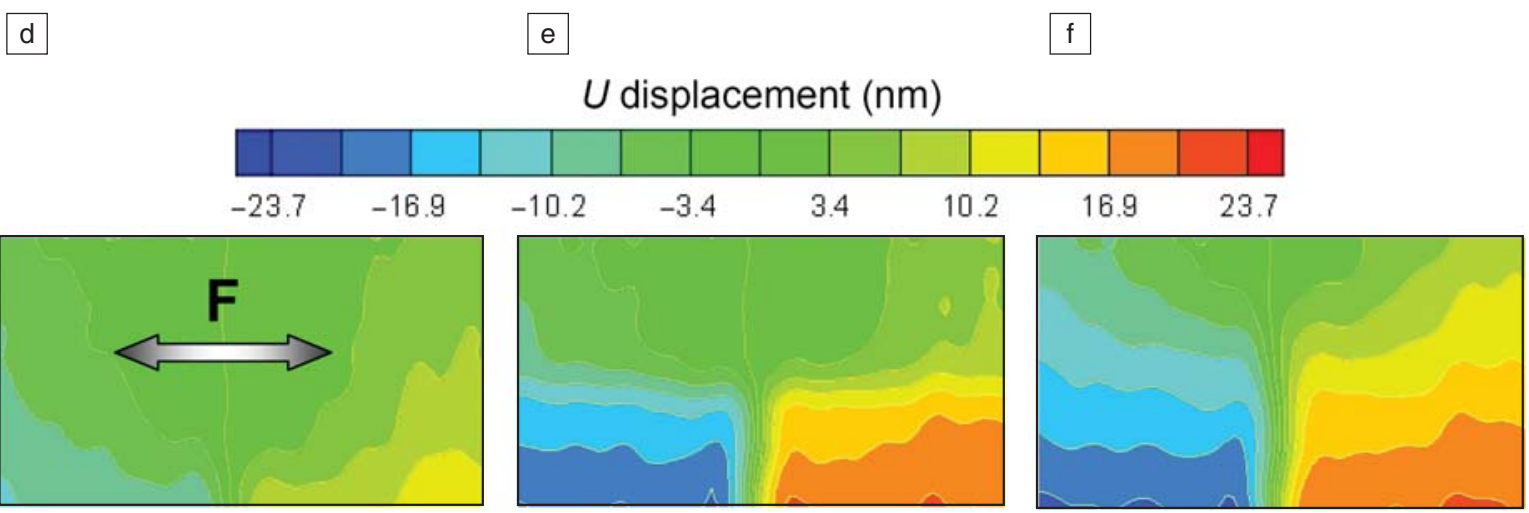

Figure 4. Subcritical crack growth in a freestanding polysilicon film. The crack propagated and arrested at distinct increments of the far-field load from (a) to (c). The displacement contours (d-f) correspond to the atomic force microscopy images (a)-(c), respectively, but they span wider fields of view ( $10 \mu \mathrm{m}$ wide by $6 \mu \mathrm{m}$ high). ${ }^{42} \mathrm{~F}$ is the applied far field force, $U$ is the local displacement field in the direction normal to the crack plane, and $K_{1}$ is the effective stress intensity factor. 


\section{In Situ Scanning Probe Microscopy Nanomechanical Testing}

a

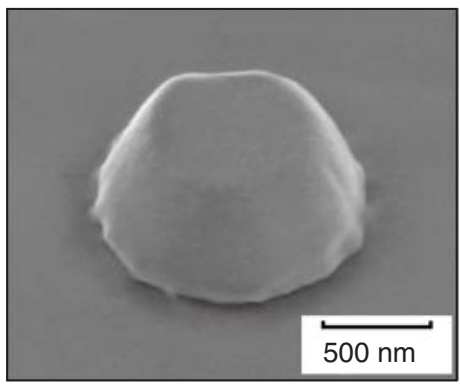

b

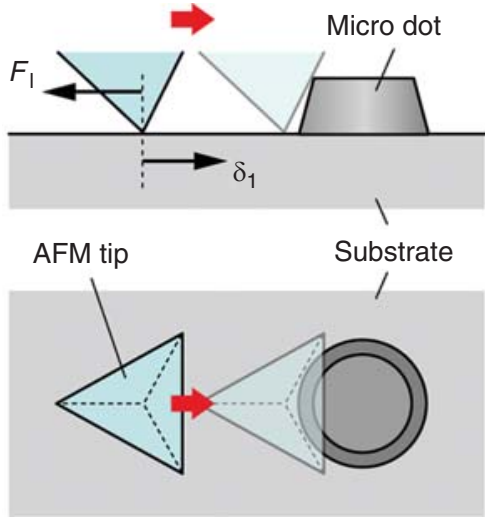

c

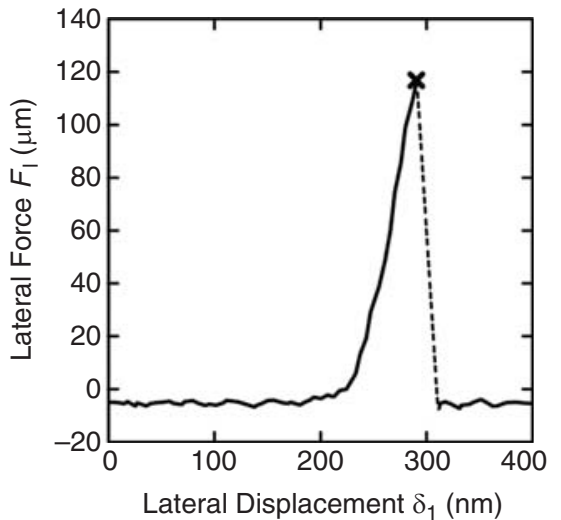

Figure 5. (a) Scanning electron microscopy image of a circular truncated cone-shaped chromium dot. (b) Schematic of an atomic force microscopy (AFM) tip dragging over the dot. The red arrow shows the direction of AFM tip motion, while $F_{1}$ is the lateral force experienced by the AFM tip. (c) The corresponding force $\left(F_{1}\right)$-lateral displacement $\left(\delta_{1}\right)$ curve. ${ }^{47}$

a hard material (e.g., W) was deposited at the top of the soft dot to constrain its surface. This allowed the dot to be successfully delaminated at its interface. ${ }^{48}$

The stress and strain distributions in the specimen at the AFM tip contact area are very complex, but their specific distribution is not sensed sufficiently away from the tip contact point. The details of the perturbation in an elastic field are discernible only at small distances from the point of perturbation. When the interface in a nanostructure is studied, it is suggested that the AFM load be applied far away from the interface, as shown in Figure 6. In such experiments, the interfacial fracture was recorded in conjunction with imaging by transmission electron microscopy. ${ }^{49}$ This method can be extended to study the creep and fatigue properties of nanostructures.
Another example that takes advantage of AFM's 2D loading capability to study the vertical and lateral rigidities of a thin film comprised of $\mathrm{Ta}_{2} \mathrm{O}_{5}$ helical nanosprings is illustrated in Figure 7.50 The apparent Young's modulus and the shear modulus of the $\mathrm{Ta}_{2} \mathrm{O}_{5}$ nanospring thin film were measured to be two to three orders of magnitude smaller than those of a conventional solid thin film of the same material. It is possible to "design" the mechanical properties of the nanospring thin film by selecting appropriate materials and by controlling the shape and size of the nanosprings.

\section{Summary}

This review article identified important contributions of scanning probe microscopy (SPM) to the mechanical testing of nanostructures and nanostructured bulk materi- als. SPM combines the capabilities of nanoscale high spatial resolution imaging, actuation, and sensing, thus making in situ mechanical testing possible and easier than other microscopy imaging tools. In addition, SPMs do not involve electron beam radiation and vacuum, which greatly affect the mechanical behavior of nanostructures and biomaterials. With other functionalities, such as the measurement of magnetic fields, electric potentials, capacitance, current flow, piezoelectric response, and temperature, SPM can provide correlations between mechanical properties and other physi$\mathrm{cal} / \mathrm{chemical}$ properties. The integration of SPMs with other imaging tools has generated new opportunities for studying material deformation and fracture mechanisms and their correlations with crystal structure defects (e.g., dislocations and twins) of nanostructures. We anticipate a
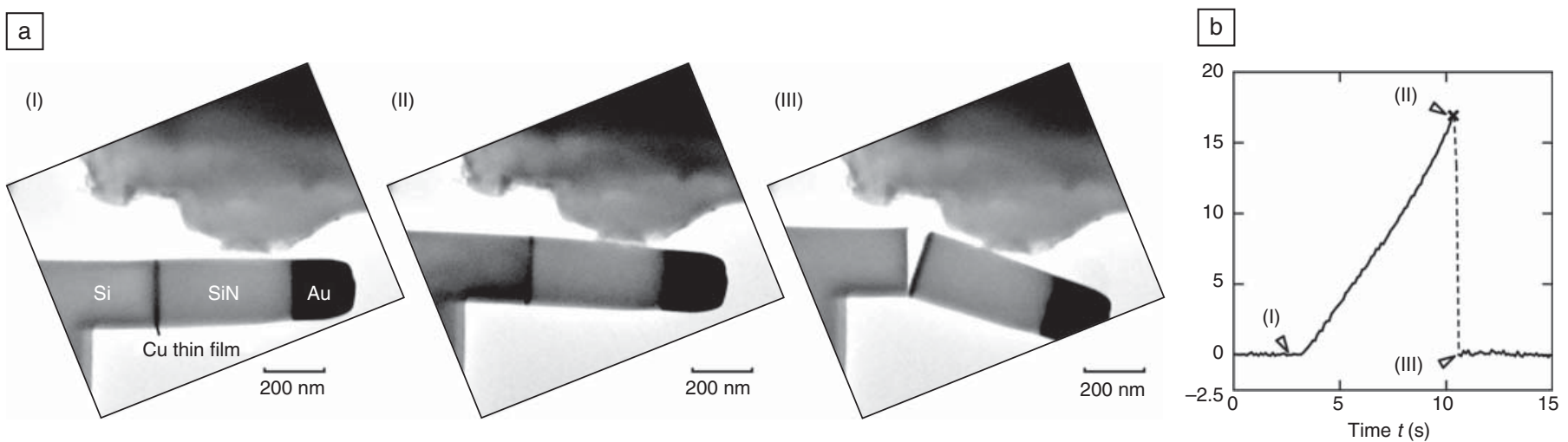

Figure 6. (a) Transmission electron microscopy micrographs of the fracture of the Si/SiN interface in a nanoscale cantilever. Stage I: the atomic force microscopy (AFM) tip approaches the nanocantilever surface. Stage II: the AFM tip loads on the SiN and bends the nanoscale cantilever. Stage III: fracture occurs at the Si/SiN interface. (b) AFM applied force as a function of time in the corresponding three stages in (a). ${ }^{49}$ 


\section{In Situ Scanning Probe Microscopy Nanomechanical Testing}

a
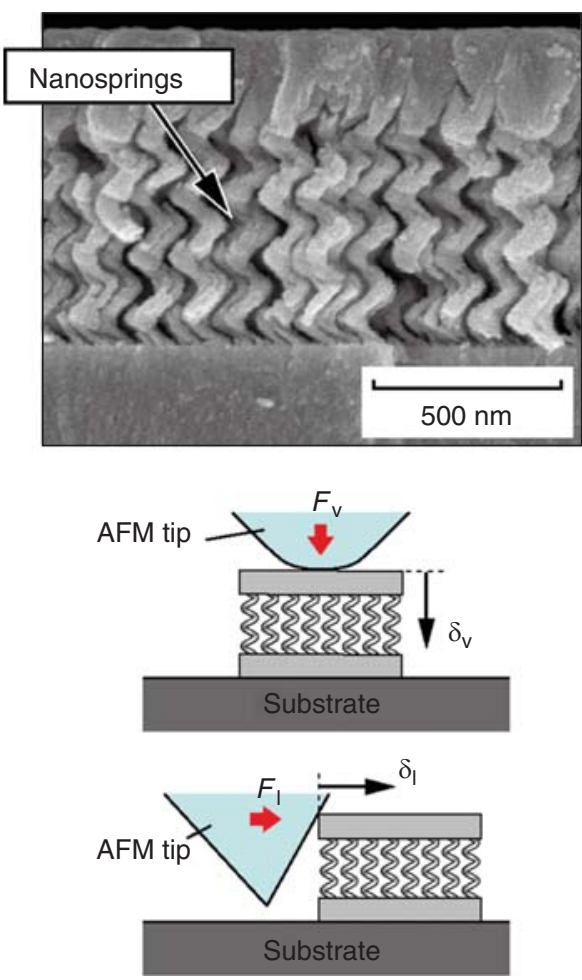

b
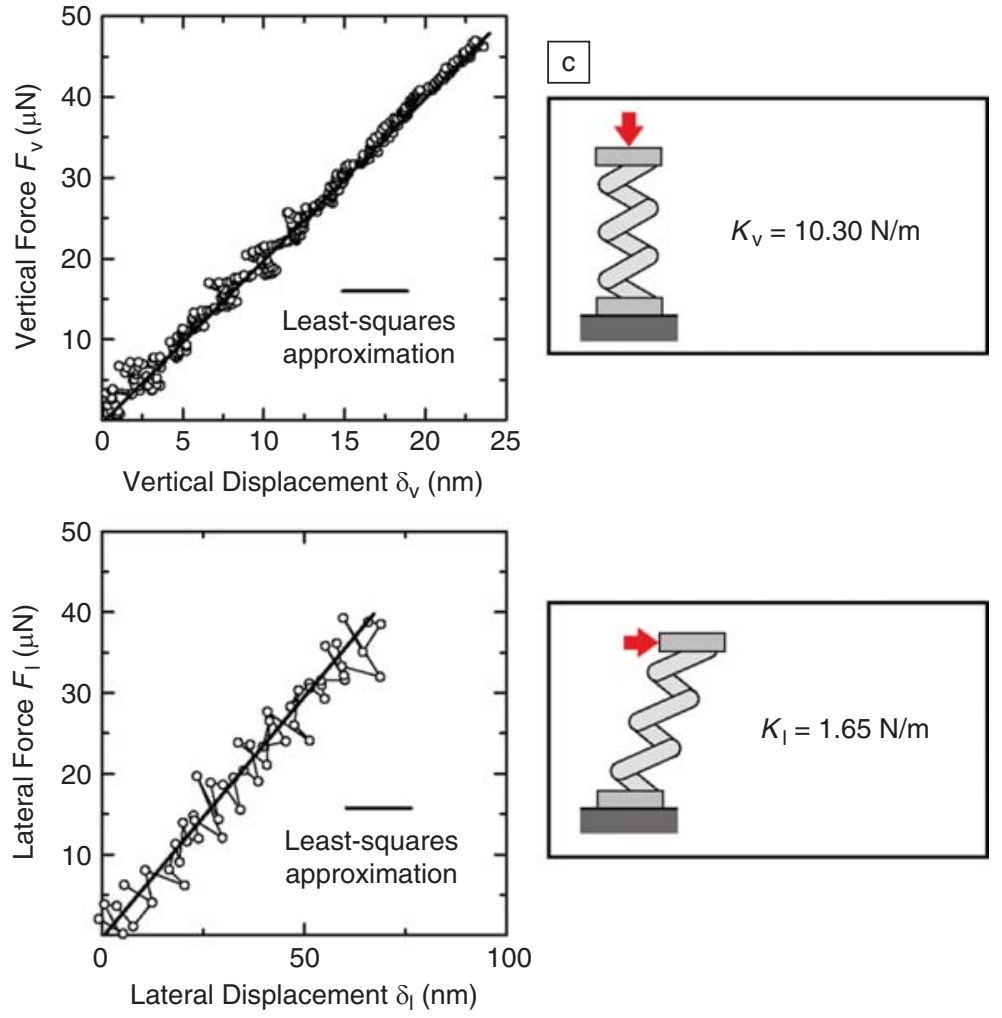

Figure 7. (a) Two-dimensional loading configurations on a thin film comprised of $\mathrm{Ta}_{2} \mathrm{O}_{5}$ helical nanosprings. $F_{\mathrm{v}}$ is the vertical force, and $\delta_{\mathrm{v}}$ is the vertical displacement; $F_{1}$ is the lateral force, and $\delta_{1}$ is the lateral displacement. (b) Examples of the vertical and lateral load-displacement curves. (c) Schematics of the nanospring thin film and the measurement of the vertical and the lateral stiffness..$^{50}$ AFM is atomic force microscopy, $K_{v}$ is the vertical spring constant, and $K_{l}$ is the lateral spring constant.

that SPM will continue to play a key role in the growing development of nanomechanical testing instruments and in the study of mechanical property size effects in nanomaterials.

\section{Acknowledgments}

X.L. acknowledges support from the National Science Foundation (CMMI0653651 and CMMI-0824728), the Army Research Office (W911 NF-07-1-0449 and W911NF-08-0299), and the ACS Petroleum Research Fund (ACS PRF 40450-AC10). I.C. is grateful for the support from the Air Force Office of Scientific Research (FA9550-06-1-0140), the National Science Foundation (DMI-0532320), and the Office for Naval Research (N00014-07-1-0888). T.K. thanks the support from the Japan Society of the Promotion of Science via a Grant-in-Aid for Scientific Research (S)(N0.21226005).

\section{References}

1. X.D. Li, B. Bhushan, Mater. Charact. 48, 11 (2002).

2. X.D. Li, H.S. Gao, C.J. Murphy, K.K. Caswell, Nano Lett. 3, 1495 (2003).
3. X.Y. Tao, X.D. Li, Nano Lett. 8, 505 (2008). 4. X.Q. Chen, Z.H. Xu, X.D. Li, M.A. Shaibat, Y. Ishii, R. S. Ruoff, Carbon 45, 416 (2007).

5. T. Ozkan, M. Naraghi, I. Chasiotis, Carbon 48, 239 (2010).

6. M. Naraghi, I. Chasiotis, Y. Dzenis, Y. Wen, H. Kahn, Appl. Phys. Lett. 91, 151901 (2007).

7. M. Naraghi, I. Chasiotis, Y. Dzenis, Y. Wen, H. Kahn, Rev. Sci. Instrum. 78, 0851081 (2007).

8. I. Chasiotis, Atomic Force Microscopy in Solid Mechanics, Handbook for Experimental Solid Mechanics, W.N. Sharpe, Jr., Ed. (Springer, NY, 2008), pp. 409-443.

9. B.D. Huey, W. Sigmund, JOM 59, 11 (2007).

10. J.P. Salvetat, A.J. Kulik, J.M. Bonard, G.A.D. Briggs, T. Stockli, K. Metenier, S. Bonnamy, F. Beguin, N.A. Burnham, L. Forro, Adv. Mater. 11, 161 (1999).

11. J.P. Salvetat, G.A.D. Briggs, J.M. Bonard, R.R. Bacsa, A.J. Kulik, T. Stockli, N.A. Burnham, L. Forro, Phys. Rev. Lett. 82, 944 (1999).

12. E.W. Wong, P.E. Sheehan, C.M. Lieber, Science 277, 1971 (1997).

13. B. Wu, A. Heidelberg, J.J. Boland, Nat. Mater. 4, 525 (2005).

14. H. Ni, X.D. Li, H.S. Gao, Appl. Phys. Lett. 88, 043108 (2006).

15. H. Ni, X.D. Li, G.S. Cheng, R. Klie, J. Mater. Res. 21, 2882 (2006).

16. H. Ni, X.D. Li, Nanotechnology 17, 3591 (2006).
17. A. Heidelberg, L.T. Ngo, B. Wu, M.A. Phillips, S. Sharma, T.I. Kamins, J.E. Sader, J.J. Boland. Nano Lett. 6, 1101 (2006).

18. H. Ni, X.D. Li. J. Nano Res. 1, 10 (2008).

19. P. Zhou, C.W. Wu, X.D. Li, Meas. Sci. Technol. 19 (2008).

20. J.H. Song, X.D. Wang, E. Riedo, Z.L. Wang, Nano Lett. 5, 1954 (2005).

21. S. Barth, C. Harnagea, S. Mathur, F. Rosei, Nanotechnology 20, 115705 (2009).

22. M.J. Gordon, T. Baron, F. Dhalluin, P. Gentile, P. Ferret, Nano Lett. 9, 525 (2009).

23. M. Lucas, W.J. Mai, R.S. Yang, Z.L. Wang, E. Riedo, Nano Lett. 7, 1314 (2007).

24. M. Lucas, W.J. Mai, R.S. Yang, Z.L. Wang, E. Riedo, Philos. Mag. 87, 2135 (2007).

25. X.D. Li, W.C. Chang, Y.J. Chao, R.Z. Wang, M. Chang. Nano Lett. 4, 613 (2004).

26. X.D. Li, Z.H. Xu, R.Z. Wang, Nano Lett. 6, 2301 (2006).

27. X.N. Wang, Z.W. Niu, S.Q. Li, Q. Wang, X.D. Li, J. Biomed. Mater. Res. Part A 87A, 8 (2008).

28. J.H. Rong, F. Oberbeck, X.N. Wang, X.D. Li, J. Oxsher, Z.W. Niu, Q. Wang. J. Mater. Chem. 19, 2841 (2009).

29. G.F. Wang, X.D. Li, Appl. Phys. Lett. 91, 231912 (2007).

30. G.F. Wang, X.D. Li, J. Appl. Phys. 104, 113517 (2008). 


\section{In Situ Scanning Probe Microscopy Nanomechanical Testing}

31. G. Stan, C.V. Ciobanu, P.M. Parthangal, R.F. Cook, Nano Lett. 7, 3691 (2007).

32. I. Chasiotis, W.G. Knauss, Exp. Mech. 42, 51 (2002).

33. S. Cho, I. Chasiotis, T.A. Friedmann, J.P. Sullivan, J. Micromech. Microeng. 15, 728 (2005)

34. I. Chasiotis, IEEE Trans. Device Mater. Reliab. 4, 176 (2004).

35. S.W. Cho, J.F. Cardenas-Garcia, I. Chasiotis, Sens. Actuators, A 120, 163 (2005).

36. S.W. Cho, I. Chasiotis, Exp. Mech. 47, 37 (2007).

37. Q. Chen, I. Chasiotis, C. Chen, A. Roy, Compos. Sci. Technol. 68, 3137 (2008).

38. E. Guilloteau, H. Charrue, F. Creuzet, Europhys. Lett. 34, 549 (1996). 39. C. Marliere, S. Prades, F. Celarie, D. Dalmas, D. Bonamy, C. Guillot, E. Bouchaud, J. Phys. Condens. Matter 15, S2377 (2003).

40. S. Prades, D. Bonamy, D. Dalmas, E. Bouchaud, C. Guillot, Int. J. Solids Struct. 42, 637 (2005)

41. Y. Tanaka, Y. Kawauchi, T. Kurokawa, H. Furukawa, T. Okajima, J.P. Gong, Macromol. Rapid Commun. 29, 1514 (2008).

42. I. Chasiotis, S.W. Cho, K. Jonnalagadda, J. Appl. Mech. Trans. ASME 73, 714 (2006).

43. S.W. Cho, K. Jonnalagadda, I. Chasiotis, Fatigue Fract. Eng. Mater. Struct. 30, 21 (2007).

44. X.D. Li, W.J. Xu, M.A. Sutton, M. Mello, IEEE Trans. Nanotechnol. 6, 4 (2007).

45. X.D. Li, W.J. Xu, M.A. Sutton, M. Mello, Mater. Sci. Technol. 22, 835 (2006).

46. Z.H. Xu, M.A. Sutton, X.D. Li, Acta Mater. 56, 6304 (2008).

47. H. Hirakata, Y. Takahashi, S. Matsumoto, T. Kitamura, Eng. Fract. Mech. 73, 2698 (2006).

48. H. Hirakata, T. Kitamura, Y. Yamamoto, JSME Int. J., Ser. A 47, 324 (2004).

49. H. Hirakata, Y. Takahashi, D. Van Truong, T. Kitamura, Int. J. Fract. 145, 261 (2007)

50. T. Sumigawa, H. Hirakata, M. Takemura, S. Matsumoto, M. Suzuki, T. Kitamura, Eng. Fract. Mech. 75, 3073 (2008)

\section{J A N I S}

\section{UHV Micromanipulated} Probe Station

UVH environment for electro-optical probing of wafers, chips, nanoscale devices

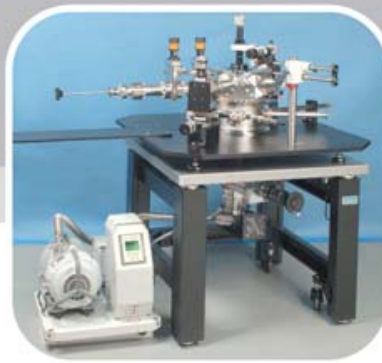

Vacuum level: $\sim 5 \times 10^{-8}$ Torr at $290 \mathrm{~K}$ and $\sim 5 \times 10^{-9}$ Torr at $5 \mathrm{~K}$

- Ultra-low vibrations ( $<2 \mathrm{~nm} / \mathrm{min}$ position drift) and wide temperature range $(4-450 \mathrm{~K})$

- Rapid sample change via load lock with proprietary demountable cryogenic sample holder

Two to six probes, DC to $67 \mathrm{GHz}$, plus fiber optics

- Complete package includes temperature controller, zoom monoscope, integrated turbopump, vibration isolation stand

\section{Janis Research Company}

2 Jewel Drive Wilmington, MA 01887 USA

TEL +1 $978657-8750$ FAX +1 978 658-0349 sales@janis.com Visit our website at WWW.janis.com

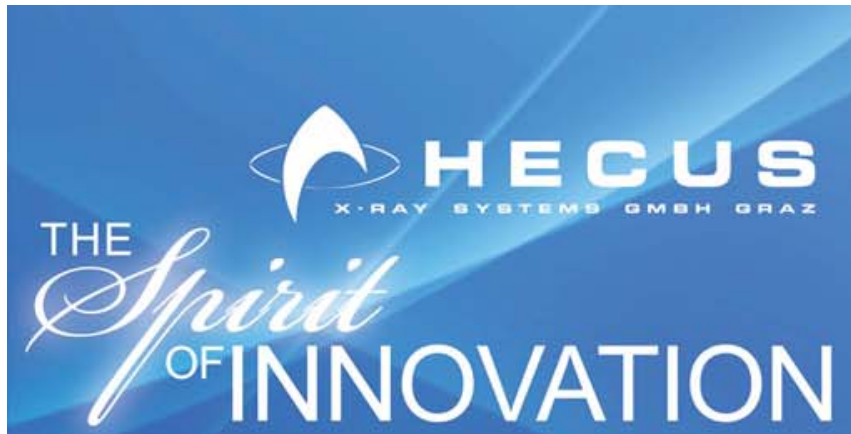

Nanostructure meets Calorimetry

\section{S3-MICROcaliX}

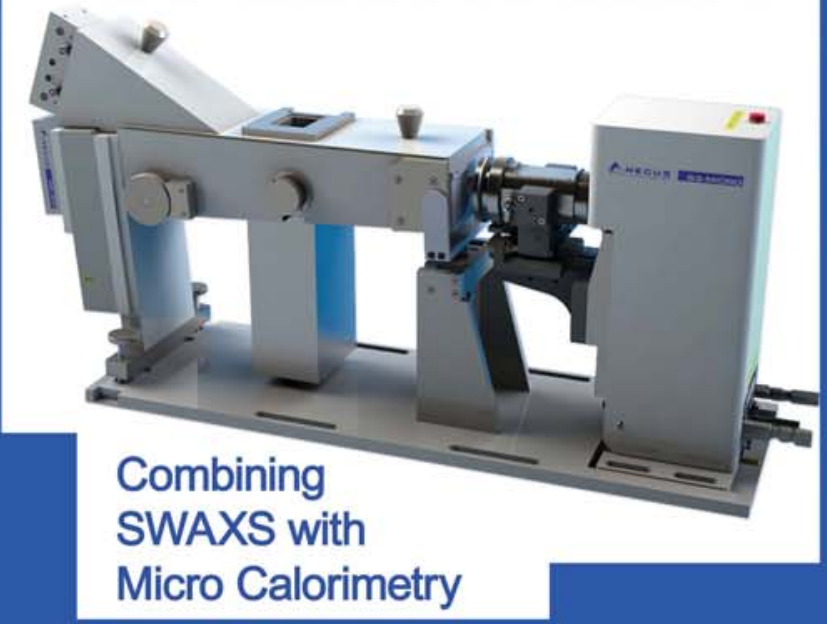

Absolute SAXS

Zero Background

Highest SAXS Resolution

\section{Unique SWAXS Instrument}

Separate, parallel-readout

Position Sensitive Detectors

Uniquely embedded CALORIMETER

Sensitivities fully matched

Direct access to sample

Unique calorimetric design

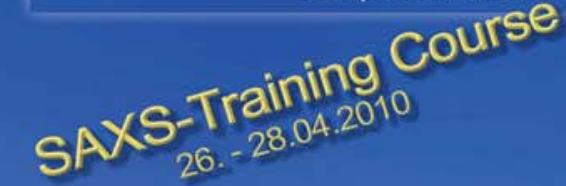

www.hecus.at 